\title{
Aspectos ultra-estruturais da fibra muscular cardíaca de ratas albinas durante o ciclo gravídico-puerperal
}

\author{
A.W. Liberatori Filho, C.D. Lopes, M. J. Simões, O.A. Mora, A.C.C. Carvalho, A.C. Lopes \\ Disciplina de Clínica Médica; Disciplina de Cardiologia do Departamento de Medicina e Disciplina de Histologia e Biol ogia Estrutural \\ do Departamento de Morfologia da Universidade Federal de São Paulo - Escola Paulista de Medicina, São Paulo, SP.
}

\begin{abstract}
RESUMO - ОвJ ETIVo. No presente estudo avaliamos, por intermédio da microscopia el etrônica detransmissão, a morfologiadoscardiomiócitosdoventrículoesquerdode ratasalbinasnodecorrer dociclogravídico-puerperal.

Métodos. Obtida a prenhez, 77 ratasforam divididas ao acasoem dois grupos denominados, respectivamente, de: gestação (G) e puerpério (P). Osani mais pertencentes ao grupo gestação foram ainda subdivididosem quatro subgrupos, de acordo com a idade gestacional; 10(G-A), 70(G-B), 14(G-C) e 21ㅇ(G-D) dias de prenhez. O grupo definido como puerpério foi também subdividido em três subgrupos; 7ㅇ (P-A), 14ㅇ (P-B) e 21ㅇ (P-C) dias de puerpério. F indo o período estabelecido para cada subgrupo, os ani mais foram sacrificados, sendo coletados fragmentos do terço médio do ventrículo esquerdo, os quais após processamento apropriado, permitiram observação adequada à mi croscopia eletrônica detransmissão.
\end{abstract}

Resultados. Os resultados obtidos demonstraram

\section{INTRODUÇÃO}

No decorrer do ciclo gravídico-puerperal ocorrem profundas alterações hormonais, metabólicas, funcionais e anatômicas em todo o organismo materno, resultando em um desvio fisiológico adaptativo de duração determinada, cuja principal finalidadeé preservar as demandas sistêmi cas necessárias ao adequado crescimento e desenvolvimento do concepto. Durante o transcurso da gestação, especificamente no sistema circulatório, ocorrem marcantes alterações no volume sangüíneo e na resistência vascular sistêmica, niti damente observadas nos primei ro e segundo trimestres da gestação. O conhecimento dos mecanismos que regem essas al ter ações hemodi nâmi cas adaptativas do sistema cardi ovascular materno durante o ciclo gravídico-puerperal ainda é tema controverso na literatura, provavel mente por causa da plural idade de resultados oriundos de diferentes técnicas de investigação.

No que se refere ao tamanho, forma e posição do coração no cicl o gravídi co-puerperal, os autores, em geral, concordam que a área cardíaca aumenta du- hi pertrofia gradativos dos cardiomiócitos nodecorrer da prenhez (aumento das mi ofi brilas, que ao final da gestação apresentaram-seentremeadas denumerosas mitocôndrias). As paredes dos capilares sangüíneos, progressivamente, tornaram-se mais delgadas, com aumento das vesículas de pinocitose no interior das células endoteliais. Revelou ainda, acentuadas dobras dosarcolema em nível dostúbulos (túneis capilares). Nogrupo puerpério, notamos gradativa regressãonessas alterações, com retorno às características prégestacionais. Osachados demonstram a ocor rência de hipertrofia no decorrer da gestação.

Conclusão. Durante a prenhez há hi pertrofia em cardiomióci to do ventrículo esquer do com regressão durante o puer pério.

UNITERMOS: Coração. Gravidez. Hipertrofia ventricular esquerda. Adaptação fisiológica. Ultra-estrutura. Cardiomiócito.

rante a gestação. Discutem, no entanto, se o fato deve-seà hi per tr ofia do múscul o cardíaco ou somente ocorre em conseqüência das al ter ações de posi ção e forma do coração, causadas pelas modificações estáticas e dinâmicas da caixa torácica, determinadas pel o crescimento progressivo doúterogravídico.

As observações vêm de longa data. Em 1857, ${ }^{*}{ }^{1}$ L archer formulou a hipótese da hipertrofia do ventrículo esquerdo durante a gestação normal. Esse ponto de vista permaneceu até que ${ }^{* 2}$ Müller e *3Drysel , respectivamente, em 1883 e 1891, concluíram que a hipertrofia ventricular na gravidez normal, quando presente, era proporcional ao peso corpóreo, descaracterizando assim um possível estado patológico.

E m 1921, Mackenzi $e^{1}$ acr editava que as variações da área cardíaca, observadas durante a gestação fisiológica, poderiam resultar das modificações da posição e da forma do coração dentro da caixa torácica, decorrentes de modificações anatômicas próprias do período.

*11 LARCHER apud Modificações gerais do organismo materno. In: BRIQUET, R.A. - Obstetrícia Normal. 3. ed. São Paulo, Savier, 1987. p. 139-74; *2 MÜLLER apud Modificações gerais do organismo materno. In:

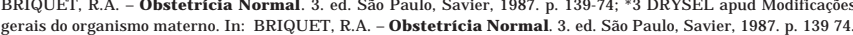


Em 1941, Hamilton e Thomson ${ }^{2}$ notaram que incrementos significati vos na área cardíaca, durante o transcurso do período gestacional fisiológico, relacionava-se com significativas alterações dos diâmetros transversal e longitudinal do tórax. Assim, inversamente, a imagem cardíaca, quando inalterada, associava-se à pequenas variações nesses diâmetros.

Somente após o advento da ecocardiografia transtorácica que, por tratar-se de técnica semiológica inócua e não-invasiva, foi possível acompanhar as alterações funcionais e anatômicas do sistema cardiovascular materno no evoluir do ci clo gravídico-puerperal. Nessesentido, esob essa nova perspectiva, Rubler, Pradohkumar, Pinto ${ }^{3}$; Katz, Karliner, Resnik ${ }^{4}$; Laird-meter et al. ${ }^{5}$ avaliaram gestantes normais pela ecocardiografia unidimensional, demonstrando aumento da massa e da espessura da parede ventricular esquerda a partir da $12^{a}$ semana. Tais achados permitiram concluir que o processo de hipertrofia excêntrica, presente no período gestacional, seria um mecanismo compensador de dilatação ventricular induzida pela sobrecarga vol umétrica, vi sando diminuir a tensão da parede miocárdica e preservar o desempenho contrátil do coração.

Lopes et al. ${ }^{6}$, avaliando os aspectos ul tra-estruturais do mi ocárdio de ratas prenhas, demonstraram aumento progressivo na concentração mitocondrial; evi denciaram também redução de sua matriz e conseqüente agl utinação de suas cristas no decurso da prenhez. Concluíram que os achados constituem o substrato ultra-estrutural do aumento do metabol i smo oxi dati vo aeróbi o da fibra cardíaca no período.

Limacher ${ }^{7}$, ao considerar as al terações do sistema cardiovascular materno, demonstrou que a hipertrofia excêntrica do ventrículo esquerdo desempenhava papel essencial no mecanismo fisiológico adaptativo frente à sobrecarga volumétrica induzida pela gravidez, ou seja, por definição, a dilatação da câmara cardíaca faz-se mantendo a espessura miocárdica. Assim, determinaria aumento da massa muscular cardíaca.

Em 1996, Liberatori Filho ${ }^{8}$ demonstrou, baseado em seus achados morfológicos e morfométricos, a ocorrência de hi pertrofia dos cardiomiócitos oriundos do ventrícul o esquerdo de ratas al binas durante a prenhez.

No que diz respeito ao período puer peral, aceitase que as alterações adaptativas desenvolvidas no decorrer da gestação progressivamente tendam a desaparecer, restabelecendo, assim, as condições funcionais e anatômicas pré-gestacionais ${ }^{9}$.

Ao analisarmos os inúmeros trabalhos existen- tes na literatura, pudemos notar que há controvérsia em se considerar a existência de um processo dinâmico e reversível de remodel ação ventricular, em conseqüência das al terações fisiológi cas adaptativas do sistema cardiovascular materno no decorrer do período gestacional normal. Apesar de constatarmos que a grande maioria dos autores acredita na ocorrência de hipertrofia ventricular durante a gestação, embasados em achados radiológi cos e ecocardiográficos, propusemos estudar, por meio da microscopia el etrônica de transmissão, os cardiomiócitos do ventrículo esquerdo de ratas al bi nas durante o ciclo gravídico-puerperal .

\section{MATERIAL E MÉTODO}

F oram utilizadas ratas (Rattus norvegi cus al binus, Rodentia, Mammalia), adultas, com idade variando entre 90 a 120 dias, virgens, pesando entre 240 e 280 gramas, provenientes da li nhagem OUTB EPM-1 (Wistar) Br Epm (2C), procedentes do CEDEME - Centro de Desenvol vi mento de Model os Experimentais em M edicina eBiol ogia da U niversidade Federal de São Paulo - Escola Paulista de Medicina (UNIFESP-EPM).

Os animais foram acondicionados em gaiolas e manti dos sob condições ideais, tendo al imentação e água ad libitum. Findo o período de adaptação, os animais foram acasalados na proporção de duas fêmeas para cada macho. A determinação da prenhez foi obtida por meio da pesquisa de espermatozóides na vagina ${ }^{10}$. O teste, quando positivo, foi considerado como o primeiro dia de prenhez.

Obti do o acasal amento, 77 animais foram di vididos ao acaso em dois grupos: gestação (G) e puerpéri o (P). Os animais pertencentes ao grupo gestação foram ainda subdividi dos em quatro subgrupos, de acordo com a idade gestacional: $1^{\circ}$ (G-A), $7^{\circ}$ (G$B), 14^{\circ}$ (G-C) e $21^{\circ}(\mathrm{G}-\mathrm{D})$ dias de prenhez. O grupo definido como puer péri o foi também subdi vidido em três subgrupos: $7^{\circ}(\mathrm{P}-\mathrm{A}), 1^{\circ}(\mathrm{P}-\mathrm{B})$ e $21^{\circ}(\mathrm{P}-\mathrm{C})$ dias do período puerperal.

Salientamos que as ratas do grupo gestação, excluindo-se as pertencentes ao primeiro dia de prenhez, quando sacrificadas, apresentavam sítios de implantações e fetos de acordo com a idade gestacional em que o animal se encontrava. Essa observação foi utilizada como critério determinante da inclusão da mesma no experimento.

Deve ser mencionado, ainda, que as ratas pertencentes ao grupo puer pério permaneceram acondicionadas em gaiolas individuais acompanhadas de suas respectivas crias até o momento do sacrifício, condição fundamental para a manutenção do estado puerperal. 


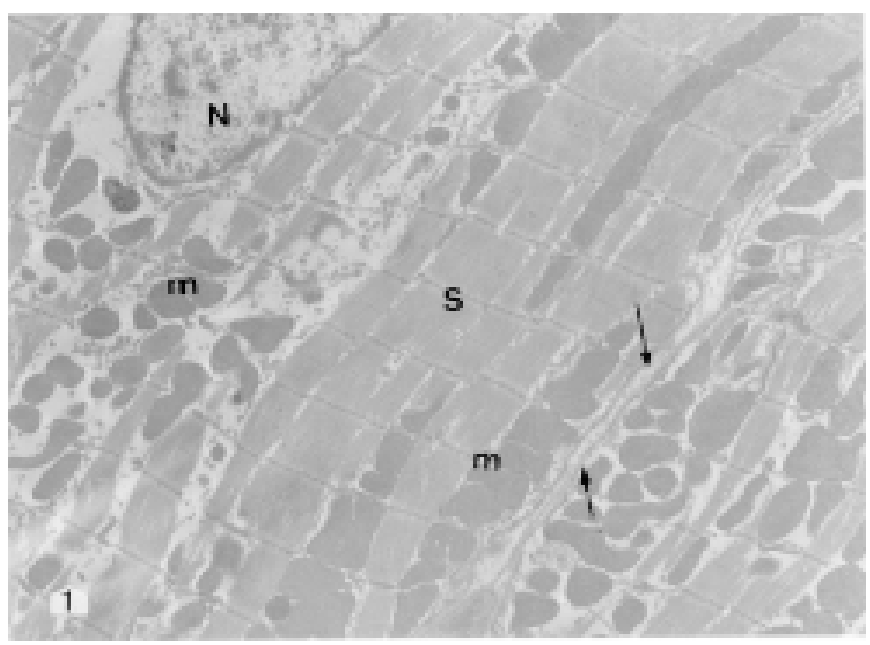

Fig. 1 - Eletromicrografia mostrando partes de dois car di omi óci tos do ventrícul o esquer do derata no 1으 (G-A) dia deprenhez. N otar doi s cardiomi óci tos separad os pel o sarcolema (setas). Observar ainda parte do núcleo (N) e mi ocôndrias (M) entremeando os sarcômeros (S) (Aumento: $7.000 \mathrm{X})$.

No período estabelecido para cada subgrupo, os animais foram anestesiados por inalação de éter etílico, assim foi possível executarmos a toracotomia, e procedermos a retirada do coração em bloco. O material coletado foi prontamente acondicionado sobre uma placa de parafina, permitindo, assim, com auxílio de uma lâmina de bisturi no 20, a obtenção de fragmentos do ter ço médi o do ventrículo esquerdo, os quais foram destinados a processamento adequado, segundo a metodol ogia descrita em literatura ${ }^{11,12}$, visando posterior observação à microscopia eletrônica de transmissão. A análise das eletromicrografias foi realizada em microscópi o el etrônico da marca Carl Zeiss, model o E M 900.

O protocolo de pesqui sa desta tese foi submetido à apreciação da Comissão de É tica Médica do Hospital São Paulo da Universidade Federal de São Paulo - Escola Paulista de Medicina, tendo sido aprovado sem ressalva.

\section{RESULTADOS}

\section{Grupo Gestação}

No 1 -dia de prenhez (G-A), as fibras cardíacas, de manei ra geral, apresentavam pequeno diâmetro e mostravam na porção periférica, plasmalema bem regular com mi ofibrilas em seu interi or, separadas por inúmeras mitocôndrias. As mitocôndrias eram, em geral, ovói des, bem regulares, contendo cristas em seu interior. Entre as mitocôndrias notamos gotículas li pídicas e corpos el etrodensos. J á, entre os cardiomiócitos, havia amplos espaços onde se visibilizavam fibrilas colágenas e capilares sangüíneos do tipo contínuo, contendo algumas vesículas de pinocitose no interior das células endoteliais (Fig. 1).

No 70 dia de prenhez (G-B), notamos que as fibras cardíacas do ventrículo esquerdo achavam-se mais desenvol vidas do que no i nício da prenhez ( 1 o dia). Apresentavam núcleo volumoso, de forma elíptica, rico em eucromatina e com nucléolo evidente. 0 citoplasma era rico em mi ofi brilas que se encontravam separadas de grande concentração de mi tocôndrias. E $m$ al gumas fibras cardíacas, havia na periferia logo abaixo do plasmalema, mitocôndrias, nesses locais denotaram-se reentrâncias da membrana celular em direção às miofibrilas em nível das linhas Z. Registramos ainda diminuição dos espaços intercelulares onde se local izavam fibrilas colágenas e inúmeros capilares sangüíneos do tipo contínuo.

No 14ㅇa dia de prenhez (G-C), observamos cardiomiócitos com núcl eo rico em eucromatina, nucl éol o conspícuo e poros nucleares bem evidentes. No citoplasma, verificamos grande concentração de miofibrilas, separadas de inúmeras mitocôndrias, repletas de cristas. $\mathrm{Na}$ porção periférica da fibra cardíaca, notamos ainda a presença de inúmeras mitocôndrias e invaginações da membrana plasmática que ocorriam regularmente em nível da linha Z dos sarcômeros, denomi nadas túneis capilares. E ntre os cardiomi ócitos foram visíveis capilares sangüíneos do tipo contínuo, com parede adelgaçada, contendo inúmeras vesículas de pinocitose e fibrilas colágenas.

No 21ㅇdia de prenhez (G-D), os cardiomiócitos eram bastante parecidos com os observados no grupo anterior (G-C), ou seja, apresentavam-se volumosos, com núcl eo rico em eucromatina, poros nucleares bem evidentes e com inúmeras mitocôndrias entre as mi ofibrilas. Na porção periférica da maioria das fibras cardíacas, havia inúmeras mi tocôndrias e invaginações da membrana plasmática que ocorriam em nível da linha $Z$ dos sarcômeros. As paredes dos capilares sangüíneos apresentavam-se nitidamente adel gaçadas, contendo inúmeras vesículas de pinocitose (Fig. 2).

\section{Grupo Puerpério}

No 70 dia de puerpério (P-A), os cardiomiócitos mostravam-se volumosos, com núcleos ricos em eucromatina, nucléol os e poros nucl eares bem evidentes. Na porção periférica de algumas fibras cardíacas, nota-seainda invaginações do sar col ema em conformidade com as linhas Z dos sarcômeros. Entre as miofibrilas, notamos inúmeras mitocôndrias ricas em cristas, al gumas com espaços el etro- 


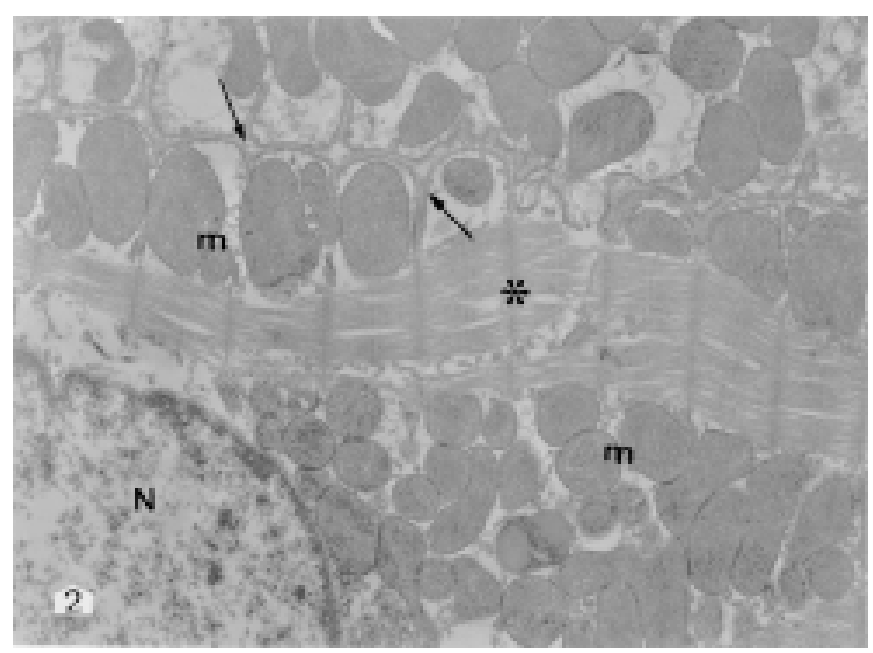

Fig. 2 - Eletromi crografia mostrando partes de dois cardi omi óci tos do ventrículo esquerdo derata no 21 ․ (G-D) dia de prenhez. Observar parte do núcl eo rico em eucromatina $(\mathrm{N})$ e inúmeros mi ofilamentos $(*)$ separadas de grande concentração de mi tocôndrias (M). Notar ainda invaginações do sarcolema em nível das linhas $Z$ dos sarcômeros (setas) (Aumento: 14.400X).

translucentes em seu interior. Observamos ainda que os espaços inter cel ulares mostravam-se discretamente aumentados, quando comparados ao grupo anterior.

No 14ㅇa dia de puerpério (P-B), observamos queas fibras car díacas do ventrícul o esquer do revel aramse menos volumosas, com tendência ao aspecto fusiforme, mostrando núcleos centrais e ricos em eucromatina. Notamos, ainda, inúmeras mitocôndrias de permei o às mi ofi brilas. Na porção periférica havia nítida retificação do sarcolema. N este grupo, os vasos sangüíneos mostravam-se circundados de inúmeras fibrilas colágenas.

No 21ㅇdi a de puer pério ( $\mathrm{P}-\mathrm{C})$, observamos que os cardiomiócitos presentes no ventrículo esquerdo possuíam forma fusiforme, eram irregulares, com núcleos elípticos, centrais e mais heterocromáticos. As fibras car díacas possuíam pequeno diâmetro, apresentavam espaços intercelulares aumentados, onde se observavam fibrilas de colágeno. A membrana plasmática mostrava-se mais retilínea que nos grupos anteriores (P-A e P-B). Os mi ofilamentos, apesar de separados uns dos outros por mitocôndrias eram, em geral, ovóides e ricos em cristas; apresentavam-se nitidamentemais organizados, conferindo um aspecto condensado aos cardi omiócitos. As paredes dos vasos sangüíneos mostravam-se delgadas, tornando o aspecto eletrodenso (Fig. 3).

\section{DISCUSSÃO}

As alter ações fisiológi cas adaptativas quesurgem

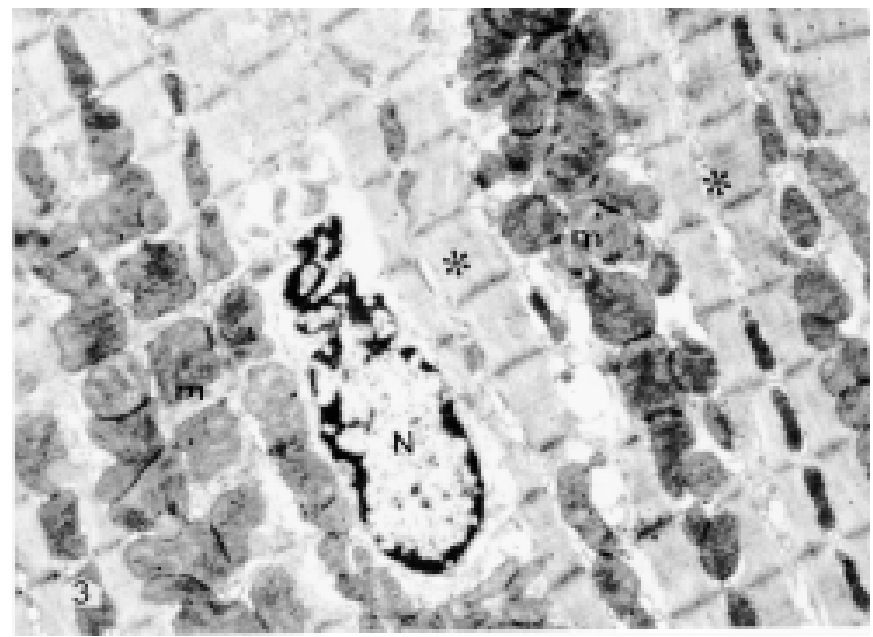

Fig. 3 - El etromi crografia mostrando partedecardiomi óci to do ventrícul o esquer do derata no 21 ㅇ (P-C) dia de puerpério. Observar núcleo heterocromático $(N)$ e mitocôndrias (M) entreos mi ofilamento $(*)$ (Aumento: $9.800 \mathrm{X})$.

no organismo materno durante o ciclo gravídicopuerperal possuem características peculiares, cuja finalidade principal é propiciar o bem-estar materno-fetal. O sistema cardiovascular materno, principalmente nos primeiros e segundos trimestres da gestação, apresenta ajustes fisiológicos em conseqüência da sobrecarga hemodinâmica determinada pel o aumento da volemia sangüínea, pela queda da resistência vascular sistêmica e pelas importantes alterações hormonais próprias do período ${ }^{13}$.

A análise da literatura, a partir do trabalho inicial de ${ }^{*}{ }^{1}$ Larcher, demonstra haver controvérsias no que diz respeito à possível ocorrência de hipertrofia do coração durante a puerperalidade. Vários autores admitiram a ocorrência de tal processo, porém seus estudos fundamentaram-se excl usivamente em análi se de i magens cardíacas (radiografia de tórax e ecocardiografia) durante o período gestacional ${ }^{14,3-5}$.

E m 1996, Liberatori Filho ${ }^{8}$ demonstrou de forma convincente, por meio de estudos morfológicos e morfométricos, a ocorrência de hipertrofia dos cardiomiócitos do ventrículo esquerdo de ratas albinas durante a prenhez. Tais resultados chamama atenção para uma característica peculiar do coração. Durante o período fetal, e até três a quatro meses após o nascimento, a hipertrofia do miocárdio faz-se às custas da hiperplasia dos cardiomiócitos. Findo esse período, o aumento da massa muscular do ventrículo que se verifica da infância à idade adulta é resultado, então, da hipertrofia celular ${ }^{15}$. O fenômeno seria responsável pelos nossos resultados. 
O coração materno frente à sobrecarga mecânica decorrente do aumento da volemia induzida pela gestação, adapta-se à mesma pelo processo de hipertrofia muscular, permitindo a normalização da tensão parietal. Esta é explicada pela Lei de Laplace ( $T=\pi . R / 2 . H$, onde: $T \rightarrow$ representa a tensão da parede, $\pi \rightarrow$ constante, $\mathrm{R} \rightarrow$ o raio da câmara e $\mathrm{H} \rightarrow$ a espessura da parede) ${ }^{16}$.

No presente trabalho, a rata foi o animal escol hido por ser habitualmente utilizada neste tipo de experimento e por permitir que os resultados obtidos possam ser mel hor comparados aos da literatura.

O estudo subcelular dos cardi omiócitos, realizado pela microscopia el etrônica de transmissão, demonstrou que durante a gestação as células cardíacas sofrem alter ações progressivas atingindo o máximo entre os 140 (G-C) e 210 (G-D) dias de prenhez (Fig. 2), retornando ao normal no final do puerpério (Fig.3). As características normais encontradas no início da gestação (Fig. 1), tais como car di omi ócitos pequenos, fusiformes, irregulares e com amplos espaços intercelulares cedem lugar a cardiomiócitos volumosos com núcleo rico em eucromatina, capil ares sangüíneos com paredes adel gaçadas contendo incontáveis vesículas de pinocitose e diminui ção i mportante do espaço intercelular (Fig. 2).

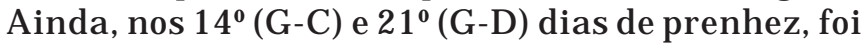
detectado no citoplasma dos cardiomiócitos um aumento da concentração de mi ofi brilas, separadas de inúmeras mitocôndrias que, por sua vez, mostravam-se repletas de cristas (Fig.2). Resultados semel hantes foram obtidos por Lopes et al. ${ }^{6}$, que relaci onaram esse achado ao incremento do metabol ismo oxi dativo aeróbico, tão necessári o frente à sobrecarga cardíaca.

Visibilizamos na porção periférica dos car diomiócitos, a partir do 140 dia de prenhez, inúmeras mitocôndrias e invaginações do sarcolema, sendo que estas últimas ocorrem regularmente em nível das linhas $Z$ dos sarcômeros (Fig. 2). As invaginações do sarcolema, denominadas túneis capilares, foram evidenciadas por Ratajska et al. ${ }^{17} \mathrm{em}$ model os experi mentais de sobrecarga vol umétrica, sendo consideradas pel os autores como achado característi co de hi pertrofia cardíaca excêntrica.

Acreditamos que essas estruturas correspondam, na realidade, às invagi nações do sarcolema, túbulos transversos ( $T$ ) que ocorrem normalmente na fibra cardíaca dos mamífer os em nível das linhas Z, e que em nosso experimento, em razão do aparecimento das mi tocôndrias logo abai xo da membrana citoplasmática, provocariam esse tipo de arranjo18. Deve ser salientadoque os túbul os T foram achados associados ao retícul o sarcoplasmático, porém não tão organizados como na fibra muscular estriada esquel ética.
É de se notar ainda que, com o decorrer da prenhez, as paredes dos capilares sangüíneos mostraram-se progressivamente adel gaçadas, contendo no interior das células endoteliais inúmeras vesículas de pi nocitose que associadas ao aumento gradativo da eucromatina nucl ear suger iram incremento do metabolismo oxidativo dos cardiomiócitos nesse período (Fig. 2).

No decorrer do puerpério, ocorreu diminuição progressiva no diâmetro dos cardiomiócitos, evidenciada indiretamente pelo aumento gradativo dos espaços intercelulares com os cardiomiócitos, voltando a apresentar suas características usuais; sendo que o aspecto mais compacto observado nessas células denotou diminuição do vol ume celular (Fig. 3). I sso mostra que as alterações cardíacas encontradas durante a gestação são rever síveis em todos os seus aspectos. Sal i entamos que os denomi nados túneis capilares mostraram-se mais evidentes, provavelmente em decorrência da diminuição do volume celular, demonstrando tratar-se na verdade de túbulos $\mathrm{T}$.

A sobrecarga hemodinâmica decorrente do aumento da vol emia ${ }^{19}$, o si stema renina-angi otensina aldosteron ${ }^{20}$, a angiotensina $\mathrm{II}^{21}$, as prostaglandinas ${ }^{22}$, a fístula arteri ovenosa ${ }^{23}$, os fatores de crescimento placentário ${ }^{21}$, a tiroxina ${ }^{24}$ e o hormônio de cresci mento ${ }^{25}$ atuam sobre os receptores cel ulares dos cardiomiócitos, determinando uma complexa resposta constituída de várias etapas e mecanismos de transduções mecânicas. Essa resposta promove a ativação das vias de sinalização intracelulares específicas, com formação de mensageiros, tais como AM Pc, 3Pi e DAG que agem sobre o núcl eo e resultam na expressão de genes fetais que codificam a síntese de proteínas contráteis e moduladoras, responsáveis pela hipertrofia dos sarcômeros. Por tratar-se de processo fi si ológi co, a unidade funcional do coração mantém seu fenótipo com características normais, com função atepeásica aumentada, ao contrário do que ocorre quando a sobrecarga é pressórica, conforme descrito por Parker e Schneider ${ }^{26}$. Não fosse assim, estaríamos diante de um processo de remodelação ventricular irreversível. A regressão da hipertrofia do ventrículo esquerdo durante o puer pério ocorre a despeito da volemia sangüínea manter-se aumentada, assim como os demais fatores já salientados. Tal fato encontra explicação na senescência placentária, tão bem estudada por Barcoft, Herkel, $\mathrm{Hill}^{27} \mathrm{em}$ coelhas. Realmente, esse órgão deixando de funcionar como uma fístula de al to débito, determina importante redução na sobrecarga hemodinâmica, permitindo-nos inferir ser a fístula placentária um elemento importante no complexo hipertrofia do 
coração na gravidez. Essas observações abrem as portas para futuras pesquisas na área.

O processo de regressão da hipertrofia do ventrículo esquerdo, de acor do com o sal ientado, provavelmente inicia-se já no fim do período gestacional $^{8}$, cujo substrato subcelular somente pode ser denotado no puerpério.

Portanto, o cor ação durantea prenhez apresenta al terações adaptati vas decorrentes de modificações hemodinâmicas próprias desse período. Contudo, por se tratar de processo fisiológico, não ocorrem mudanças permanentes na arquitetura ventricular, que poderiam deixar seqüelas e até mesmo representar um mecanismo de progressão para insuficiência cardíaca.

\section{CONCLUSÃO}

Considerando-se as condições metodológicas destetrabalho, concluímos que os achados subcel ulares mostraram haver hipertrofia dos cardiomiócitos do ventrículo esquerdo durante a prenhez, bem como sua regressão no período puerperal .

\section{SUMMARY}

\section{Ultrastructural aspects of cardiac muscle fi- bers of al bino rats during pregnancy and pu- erperal cycle}

PuRPose. In the present study we evaluated the morphology of left ventricular cardiomyocytes of albino rats during pregnancy and puerperium by means of transmission el ectron microscopy.

Methods. Oncepregnancy was confirmed, 77 rats were randomly divided in two groups, respectively, gestation (G) and puerperium ( $P$ ). The animals of the gestation group were divided into four subgroups, according to gestational age: $1^{\text {st }}(G-A), 7^{\text {th }}$ (G-B), $14^{\text {th }}(\mathrm{G}-\mathrm{C})$ and $21^{\text {st }}$ (G-D) days of pregnancy. The group defined as puerperium was divided into threesubgroups: $7^{\text {th }}(\mathrm{P}-\mathrm{A}), 14^{\text {th }}(\mathrm{P}-\mathrm{B})$ and $21^{\text {st }}(\mathrm{P}-\mathrm{C})$ days of puerperium. In the end of each established period, the animals were sacrificed and fragments of the medium third of the left ventricle were resected and routinely prepared for electron microscopy analysis.

RESULTS. Theresults obtained with transmission el ectron microscopy analysis revealed a gradative increase of the cardiomyocytes during pregnancy (increase of myofibrils, which are permeated by numerous mitochondria at the end of gestation). The blood capillary wall showed progressive thinning, with an increase of pynocytotic vesicles in endothelial cells, and intensesarcol emal fol ding at T- tubule level (capillary tunnels). In the puer- perium group, there is a progressive regression in these al terations returning to pre-gestational level at the end of the puerperium cycle. These findings indicate the occurrence of hypertrophy of cardiomyocytes during pregnancy.

CONCLUSION. Thefindings indicatetheoccurrence of hypertrophy of cardi omyocytes during pregnancy. [Rev Ass Med Brasil 1999; 45(3):199-205.]

KEY WORDS: Heart. Pregnancy. L eft ventricle hypertrophy. Physiological adaptation. Cardiomyocytes.

\section{REFERÊNCIAS BIBLIOGRÁFICAS}

1. Mackenzie, Sir J . - Heart disease and pregnancy. London, Oxford, 1921.

2. Hamilton, B.E. \& Thomson, K.J . - The heart in pregnancy. Boston, Little Brown, 1941.

3. Rubler, S.; Pradohkumar, M.D.; Pinto, E.R. - Cardiac size and performance during pregnancy estimated with echocardiography. Clin. Obstet. Gynec., 1977; 40: 534-9.

4. Katz, R.; Karliner, J.S.; Resnik, R. - Effects of natural volume overload state (pregnancy) on left ventricular performance in normal human subjects. Circulation, 1978; 58: $434-41$

5. Laird-Meeter, K.; Van De Ley, G.; Bom, T.H.; Wladimiroff, J.W.; Roelandt, J. - Cardiocirculatory adjustments during pregnancy: an echocardiographic study. Clin. Cardiol., 1979; 2: 328-32.

6. Lopes, A.C.; Sasso, W.S.; Pacheco, I.P.; Mora, O.A.; Simões, M.J . - Aspectos ultra-estruturais das mitocôndrias da fibra muscular cardíaca de ratas albinas durante a prenhez. Cienc. Cult., 1983; 35: 69-74.

7. Limacher, M.C. - Echocardiography in Pregnancy. Echocardiographic, 1986; 3: 19-32.

8. Liberatori Filho A.W. - Aspectos Morfológicos e Morfométricos do Miocárdio da Rata Albina Durante a Prenhez. São Paulo, 1996. (Tese de Mestrado - Universidade Federal de São Paulo - Escola Paulista de Medicina)

9. Robson, S.C.; Hunter, S.; Moore, M.; Dunlop, W. - Hemodynamic changes during the puerperium: a doppler and $\mathrm{M}$ mode echocardiographic study. Br. J. Obstet. Gynecol., 1987; 94: 1.028-39.

10. Hamilton, J .B. \& Wolfe, J .M. - The effect of male hormone substance upon birth and prenatal development in the rat. Anat. Rec., 1938; $70:$ 433-40.

11. Luft, J .H. - I mprovements in epoxy embedding methods. J . Biophys. Biochem. Cytol., 1961; 9: 409-14.

12. Harven, E. - Methods in electron microscopic cytology. Methods Cancer Res., 1967; 1: 37-8.

13. Liberatori Filho, A.W.; Lopes, M.D.; Lopes, A.C. - Alterações funcionais do sistema circulatório na gravidez. Rev. Bras. Clin. Terap., 1998; 24 : 123-7.

14. J ensen, F.G. \& Norgaard, E. - Studies on the funcional cardiac diseases and essential cardiac hypertrophy in normal pregnant women. Acta Obstet. Gynec. Scand., 1927; 6: 67-76.

15. Anversa, P.; Ricci, R.; Olivetti, G. - Quantitative structural analysis of the myocardium during physiological growth and induced cardiac hypertrophy: a review. J. Am. Coll. Cardiol., 1986; 7: 1.140-9.

16. Omens, J.H. \& Covell, J.W. - Transmural distribution of myocardial tissue growth induced by volume overload in the dog. Circulation, 1991; 84: 1.235-45. 
17. Ratajska, A.; Feijka, E.; Maksymowicz, M.; Gawlik, Z. Tunnel capillaries in hypertrophied myocardium of rats with aorto-caval fistula. Basic Res. Cardiol., 1993; 88: 120-9.

18. Stevens, A. \& Lowe, J S. - Contractile Cells. In: Histology. 2. ed. London, Gower Medical Publishing, 1997. p. 65-76.

19. Rovinsky, J J . \& J affin, H. - Cardiovascular hemodynamic in pregnancy: blood and plasma volumes in multiple pregnancy. Amer. J . Obstet. Gynec., 1965; 93: 1-8.

20. Hanssens, M.; Keirse, M.J .; Spitz, B. - Angiotensin II levels in pregnancy. Br. J. Obstet. Gynaecol., 1991; 98: 155-9.

21. Frohlich, E.D. - Overview of hemodynamic and nonhemodynamic factors associated with left ventricular hypertrophy. J. Mol. Cell. Cardiol., 1989; 21: 3-10.

22. Fitzgerald, D.J .; Eentmann, S.S.; Mulloy, K.; Fitzgerald, G.A. - Decreased prostacylin biosynthesis preceding the clinical manifestations of pregnancy induced hypertension.
Circulation, 1987; 75: 956-9.

23. Metcalfe, J . \& Ueland, K. - Maternal cardiovascular adjustments to pregnancy. Prog. Cardiovasc. Dis., 1974; 16: 363-74.

24. Carter, W.J .; Van Der Weijden, C.; Benjamin, W.S.; Faas, F.H. - Effect of thyroid hormone on protein turnover in cultured cardiac myocytes. J. Mol. Cell. Cardiol., 1985; 17 : 897-905.

25. Fazio, S.; Cittadini, A.; Sabatini, D.; Merola, B.; Colao, A.M.; Biondi, B.; Lombardi, G.; Saccá, L. - Evidence for biventricular involvement in acromegaly: a Doppler echocardiographic study. Eur. Heart J ., 1993; 14 : 26-33.

26. Parker, T.G. \& Schneider, M.D. - Peptide growth factors can provoke "fetal" contractile protein gene expression in rat cardiac myocytes. J . Clin. Invest., 1990; 85: 507-14.

27. Barcoft, J.; Herkel, W.; Hill, S. - Rate of blood flow and gaseous metabolism of the during pregnancy. J. Pshysiol., 1933; 77: 194-201. 